\title{
Contemporary concepts of primary dynamic facial nerve reconstruction in the oncologic patient
}

Klein, Holger Jan ; Guedes, Tiago ; Tzou, Chieh-Han John ; Rodriguez-Lorenzo, Andrés

\begin{abstract}
Transection of the facial nerve and its branches during extensive ablative procedures in the oncologic patient causes loss of control of facial mimetic muscles with severe functional and aesthetic sequelae. In such patients with advanced tumorous disease, copious comorbidities, and poor prognosis, rehabilitation of the facial nerve has long been considered of secondary priority. However, recent advances in primary facial nerve reconstruction after extensive resection demonstrated encouraging results focusing on rapid and reliable restoration of facial functions. The authors summarize 3 innovative approaches of primary dynamic facial nerve reconstruction by using vascularized nerve grafts, dual innervation concepts, and intra-facial nerve transfers.
\end{abstract}

DOI: https://doi.org/10.1097/SCS.0000000000005619

Posted at the Zurich Open Repository and Archive, University of Zurich

ZORA URL: https://doi.org/10.5167/uzh-189953

Journal Article

Published Version

Originally published at:

Klein, Holger Jan; Guedes, Tiago; Tzou, Chieh-Han John; Rodriguez-Lorenzo, Andrés (2019). Contemporary concepts of primary dynamic facial nerve reconstruction in the oncologic patient. Journal of Craniofacial Surgery, 30(8):2578-2581.

DOI: https://doi.org/10.1097/SCS.0000000000005619 


\title{
Contemporary Concepts of Primary Dynamic Facial Nerve Reconstruction in the Oncologic Patient
}

\author{
Holger Jan Klein, $M D{ }^{* \dagger}$ Tiago Guedes, MD, ${ }^{\ddagger}$ Chieh-Han John Tzou, MD, ${ }^{\S}$ \\ and Andrés Rodriguez-Lorenzo, $M D, P H D^{*}$
}

\begin{abstract}
Transection of the facial nerve and its branches during extensive ablative procedures in the oncologic patient causes loss of control of facial mimetic muscles with severe functional and aesthetic sequelae. In such patients with advanced tumorous disease, copious comorbidities, and poor prognosis, rehabilitation of the facial nerve has long been considered of secondary priority. However, recent advances in primary facial nerve reconstruction after extensive resection demonstrated encouraging results focusing on rapid and reliable restoration of facial functions. The authors summarize 3 innovative approaches of primary dynamic facial nerve reconstruction by using vascularized nerve grafts, dual innervation concepts, and intra-facial nerve transfers.
\end{abstract}

Key Words: Dual innervation, facial nerve reconstruction, facial palsy, hand and neck oncology, intrafacial nerve transfer, vascularized nerve graft

(J Craniofac Surg 2019;30: 2578-2581)

\section{BACKGROUND}

$\mathrm{T}$ ransection of the facial nerve and its branches during extensive ablative procedures causes loss of control of facial mimetic muscles with severe functional and aesthetic consequences. Oral incompetence, nasal obstruction, asymmetry at rest, lagophthalmus with potential corneal injury as well as inability to produce normal facial expressions are frequently observed sequelae reducing patients' quality of life significantly. ${ }^{1}$ Besides less frequently occurring cutaneous malignancies of the periauricular region, parotid gland cancer represents the most common extra-temporal tumor entity involving the facial nerve. While most parotid gland tumors are benign and therefore treated with facial nerve-sparing techniques, malignant tumors constitute approximately $20 \%$, of which another $20 \%$ show involvement of the facial nerve. ${ }^{2}$ Total

From the *Department of Plastic and Maxillofacial Surgery, Akademiska Sjukhuset, Uppsala University, Uppsala, Sweden; †Division of Plastic Surgery and Hand Surgery, University Hospital Zurich, Zurich, Switzerland; †Department of Plastic, Reconstructive and Craniomaxillofacial Surgery and Microsurgery Unit, Vila Nova de Gaia/Espinho Hospital Centre, Porto, Portugal; and §Faculty of Medicine, Sigmund Freud University, Vienna, Austria.

Received February 17, 2019.

Accepted for publication March 21, 2019.

Address correspondence and reprint requests to Holger Jan Klein, MD, University Hospital Zurich, Division of Plastic Surgery and Hand Surgery, Raemistrasse 100, 8091 Zurich, Switzerland;

E-mail: klein.holger@gmail.com

The authors report no conflicts of interest.

Copyright $\odot 2019$ by Mutaz B. Habal, MD

ISSN: $1049-2275$

DOI: $10.1097 /$ SCS.0000000000005619 parotidectomy with resection of the involved portion of the facial nerve is the treatment of choice when facial nerve infiltration or adherence is found intraoperatively. Invasion of the facial nerve is considered a strong indicator for poor prognosis predicting metastatic spread and recurrence. In such advanced cases, rehabilitation of the facial nerve has long been considered of secondary priority, and still today, it is controversially debated since conventional reconstruction possibilities may fall short regarding rapid functional and aesthetic recovery promising augmented quality of life to patients with poor survival. However, recent investigations demonstrated that simultaneous facial nerve repair along with oncologic tumor resections is more favorable regarding the overall outcome as compared to delayed or multi-step facial nerve reconstructioneven if postoperative radiation is performed. ${ }^{3,4}$

A large number of surgical techniques and modifications have been published for the rehabilitation of the face after nerve resection, such as nerve grafting, nerve transfers, regional muscle transposition, and free muscle transfers. Regarding primary facial nerve repair, conventional non-vascularized nerve grafting has been described as most frequent choice to bridge facial nerve gaps secondary to ablative surgery using the great auricular nerve, branches of the cervical plexus, or the sural nerve as donors. Though, the degree of functional recovery following nerve grafting may vary and might be affected by several factors including postoperative radiotherapy, length of nerve gap, wound conditions, and patient's age. Likewise, in contrast to facial palsy from nonmalignant etiologies, poor long-term prognosis, advanced patients' age, and comorbidities mandate rapid restoration of form, and function. As to that, recent advances in primary facial nerve reconstruction after extensive resection demonstrated encouraging results focusing on rapid and reliable restoration in patients with poor prognosis. The authors present hereinafter 3 innovative approaches to primary facial nerve reconstruction by using:

(1) vascularized nerve grafts,

(2) intra-facial nerve transfer, and

(3) dual innervation.

These concepts should not be regarded as 3 separate reconstructive strategies, but might rather be combined in complex facial nerve restoration depending on the type of defect (soft tissue needed or not), extent of facial nerve resection (partial/isolated defect vs complete resection), site of resection (extratemporal vs intratemporal/intracranial), and need for postoperative radiation.

\section{VASCULARIZED NERVE GRAFTS}

It was not until 1976 that Taylor and Ham used a free vascularized radial nerve graft for reconstruction of a median nerve gap hypothesizing that the local blood supply of the microenvironment is crucial for effective nerve regeneration. ${ }^{5}$ With advances in microsurgical instruments and techniques, the idea to optimize blood supply to nerve bundles gained successive importance throughout animal models and clinical settings. ${ }^{6-8}$ Vascularized nerve grafts 
demonstrated reduced endoneurial scarring by maintaining the Schwann cell population and suppressing fibroblast infiltration, thus providing an optimal nutritional environment that results in an increased rate of axonal regeneration. ${ }^{9-11}$ Accordingly, numbers of myelinated fibers, axon diameter, and myelin thickness were found significantly higher in vascularized nerve grafts than in non-vascularized ones. Further studies discovered the speed of axonal sprouting to be 3 times faster in vascularized versus non-vascularized nerve grafts. ${ }^{9}$ Consequently, the timespan of muscle denervation is minimized, and compound muscle action potential measurement following vascularized nerve grafting was found nearly equal to intact nerve-muscle entities. ${ }^{12}$ These premises adequately meet the requirements of expedient nerve regeneration in a barely vascularized, potentially radiated macro milieu as found in patients after ablative treatment.

Reconstructive surgeons frequently face combined soft tissue and facial nerve defects secondary to extensive tumor resections being ideally restored by simultaneous transfer of soft tissue along with vascularized nerves. Although different vascularized nerve grafts have been suggested in reconstruction of the facial nerve, such as the lateral femoral cutaneous nerve, the posterior cutaneous arm nerve, the sural nerve, the deep peroneal nerve, and the vastus lateralis motor nerve (VLMN), the latter option seems advantageous when harvested along with the anterolateral thigh (ALT) flap both being supplied by 1 pedicle. ${ }^{7,8,11,13,14}$ In this setting, the VLMN unites the benefits of autarkic vascularization and the inherent feature as a motor nerve itself, as investigations have demonstrated motor axons to preferentially re-innervate motor rather than sensory pathways. ${ }^{15,16}$ Two main branches of the VLMN (mean diameter $2.4 \pm 0.6 \mathrm{~mm}$ ) have been identified in anatomical studies, 1 descendent branch with an average extramuscular length of $15.1 \pm 3.9 \mathrm{~cm}$ running along with the descendent branch of the lateral circumflex femoral artery which commonly gives rise to the perforators supplying the ALT flap, and 1 oblique branch (absent in 30\%) with an average length of $8.4 \pm 4.5 \mathrm{~cm} .{ }^{1}$ Additional length can be gained by intramuscular dissection. Besides its sufficient length, the VLMN shows constant multiple branching, thus optimally qualifying for facial nerve reconstruction when several branches need to be grafted. Despite potential denervation of the vastus lateralis muscle when harvesting both VLMN branches, clinical studies demonstrated no functional limitation in knee extension after 6 months-highlighting the low donor site morbidity of the vascularized VLMN. ${ }^{17,18}$

An exemplary demonstration of vascularized nerve grafting is shown in Figure 1, where a soft tissue defect with isolated partial resection of the marginalis mandibular nerve $(\mathrm{MMN})$ is restored by
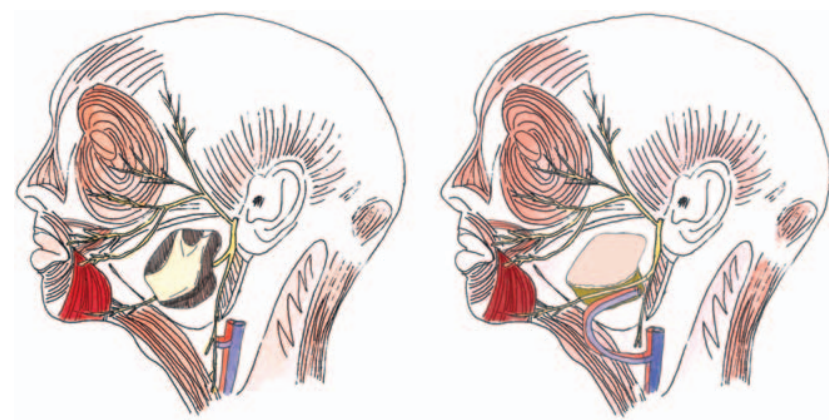

FIGURE 1. Exemplary demonstration of vascularized nerve grafting in the oncologic patient: a soft tissue defect with isolated partial resection of the marginalis mandibular branch is restored by a free ALT flap including VLMN both supplied by 1 pedicle connected to the superior thyroid vessels in the neck. $\mathrm{ALT}$, anterolateral thigh; VLMN, vastus lateralis motor nerve. a free ALT including VLMN both supplied on 1 pedicle connected to the superior thyroid vessels in the neck.

\section{DUAL INNERVATION}

Dual innervation refers in the following paragraph to the simultaneous usage of nerve transfers as well as (vascularized) autologous nerve grafts for facial reanimation after tumorous head and neck surgery providing separate innervation to 2 different functional targets of the facial nerve: eye closure (by nerve grafting the facial nerve stump to branches to the eye and forehead) and smile and orbicularis oris reanimation (by using masseter nerve transfer to zygomatic and buccal branches). While nerve grafting is only possible when the extra-temporal facial nerve stump is present, nerve transfers represent a constant autonomous source for facial reanimation. Dual innervation techniques hold the crucial advantage of a separate re-innervation of different transected facial nerve branches, thus avoiding undesirable synkinesis and increasing the axonal load in the distal branches of the facial nerve providing more reliable functional recovery.

Nerve transfers provide a healthy source of axons close to the target muscles and allow for direct coaptation leading to accelerated nerve recovery and therefore diminished duration of muscle denervation. Selection of donor nerves to-be-transferred in facial reanimation includes the hypoglossal nerve, masseter nerve, contralateral facial nerve with cross-face nerve grafts, the spinal accessory nerve, and phrenic nerve. ${ }^{19}$ Amongst those, the masseter nerve has recently increased its popularity due to its constant nerve anatomy, strength of motor impulses, fast recovery of tonus and motion, ease of cerebral adaptation, and low donor-site morbidity. $^{20-24}$ The masseteric motor nerve originates from the anterior division of the mandibular branch of the trigeminal nerve. After exiting the cranium through the foramen ovale, the nerve passes over the lateral pterygoid muscle and through the coronoid notch to enter the posterior surface of the masseter muscle near its origin. When adequately dissected, the masseteric nerve is of appropriate length to allow tension-free coaptation to facial nerve branches. With an average number of about 1600 to 2000 myelinated axons (average diameter: $1.6 \pm 0.4) \mathrm{mm}$, the masseter nerve offers sufficient cross-sectional area to connect 1 or even several branches of the facial nerve (e.g., buccal branch: 850 axons). ${ }^{21,25}$ To minimize collateral tissue damage when identifying the masseter nerve, anatomical studies defined a subzygomatic rectangle of about $1.5 \mathrm{~cm}^{2}$ - bordered by the zygomatic arch, the condyle, and coronoid process as well as the mandibular notch. ${ }^{26}$ The masseter nerve can consistently be found 10 to $15 \mathrm{~mm}$ deep to the parotidomasseteric fascia via this quick, safe, and relatively noninvasive approach. Anatomical studies demonstrated in $75 \%$ more than 1 masseteric branch innervating the masseter muscle at the level of the sigmoid notch. When transecting the masseteric nerve distal to some of these arborizing branches only partial muscle denervation is achieved. However, even full masseteric denervation demonstrated low donor site morbidity with rare occurrence of malocclusion. Of note, associated cerebral adaption rapidly allows patients to smile without biting (usually $<3$ months).

Given the presence of the facial nerve stump after extra-temporal oncologic resections, the authors prefer a dual innervation approach by:

(1) neurotizing the frontal and zygomatic branches for adequate eye function using either the (vascularized) vastus lateralis motor nerve (also see above) or the lateral femoral cutaneous nerve in order to maintain a single harvest site along with the ALT flap;

(2) masseter nerve transfer to the buccal (and marginal mandibular) branches for smile restoration and oral competence. 

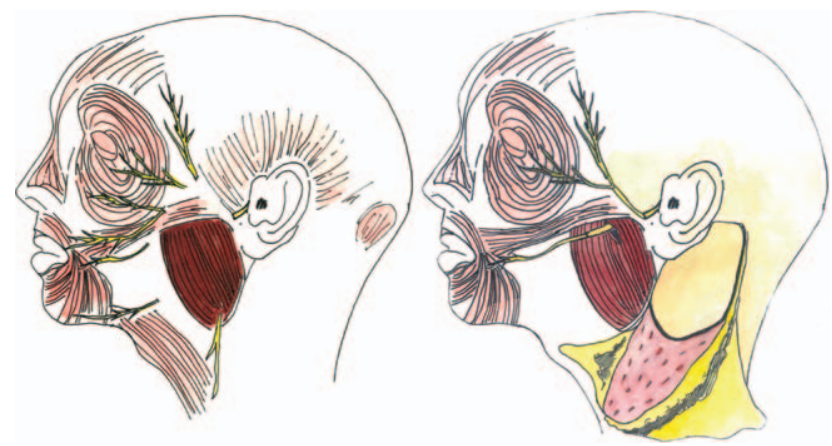

FIGURE 2. Large soft tissue defect with extensive resection of the facial nerve. Dual innervation approach by (1) neurotizing the frontal and zygomatic branches for adequate eye function using the lateral femoral cutaneous nerve in order to maintain a single harvest site along with the free ALT flap; (2) masseter nerve transfer to the buccal (and marginal mandibular) branches for smile restoration and oral competence. ALT, anterolateral thigh.

In this way, facial tonus is restored, symmetry is improved, isolated voluntary and potentially spontaneous smile is regained, and lower eyelid position and eye closure are amended. This concept of dual innervation is exemplarily shown in Figure 2.

\section{INTRAFACIAL NERVE TRANSFERS}

Besides traumatic facial nerve injuries, isolated transection of single facial nerve branches may occur during resection of malignant parotid tumors or neurolemmoma as well as neurofibroma of the facial nerve itself. Likewise, iatrogenic injury to the MMN during ablative surgery in the submandibular and parotid region can cause segmental defects, which are not amenable to tension-free end-toend anastomosis. In these scenarios, intra-facial nerve transfers provide another innovative approach to primary facial rehabilitation by redirecting less important facial nerve branches to those branches with crucial function. As to that, upper buccal or cervical branches of the facial nerve are sacrificed in order to restore zygomatic or marginal mandibular branches, thus lowering the risk for corneal exposure, drooling, and asymmetrical smile. ${ }^{27,28}$

For lack of intra-branch connections of the MMN with other facial nerve branches, spontaneous recovery of its innervated muscles is less likely in case of its damage. ${ }^{29}$ As the cervical branch (platysma motor nerve [PMN]) of the facial nerve arises from the lower division of the facial nerve coursing more posterior and deeper than the MMN, it is less prone to injury. ${ }^{27} \mathrm{MMN}$ and PMN have synergistic functions both innervating muscles expressing unhappy mimic looks, as the lower lip muscle depressors, as well as the platysma, are activated vertically from cranial to caudal. Recent anatomical findings demonstrated comfortable identification and dissection of the PMN as it runs under the platysma from the anterior-inferior part of the parotid gland. ${ }^{30}$ With an average length of about $47 \mathrm{~mm}$ and about 5 myelinated fiber counts per millimeter, the PMN qualifies optimally for direct MMN nerve repair without sacrificing further donor sites (MMN fiber counts per millimeter: 3.9). Additionally, sacrification of cervical branches did not result in clinically relevant impairment of facial movements. Similarly, upper buccal branches redirected to injured zygomatic branches cause negligible donor site morbidity since multiple communicating fibers between upper and lower buccal branches make the reappearance of the nasolabial fold likely within few months. Recent clinical findings demonstrated good to excellent outcomes of both methods described, which some authors explain by the fact of replacing "same with same." 27 In that way, facial motor nerve branches not only replace the injured motor nerve branches but also function as direct vascularized nerve grafts, which
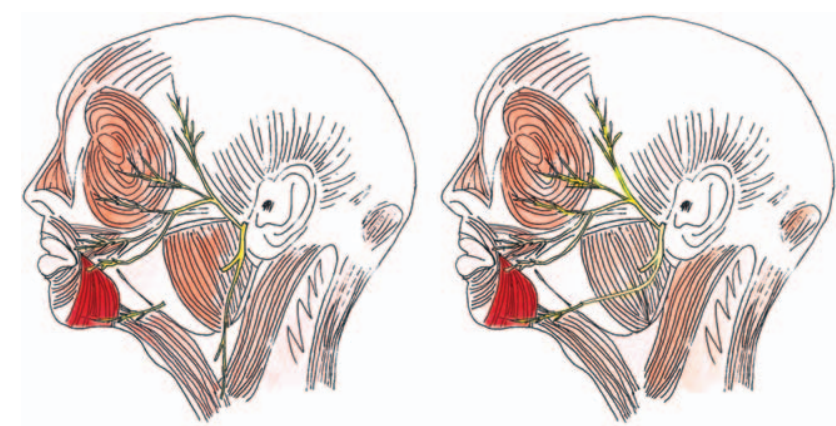

FIGURE 3. An isolated partial defect of the MMN is restored by intrafacial transfer of the PMN. MMN, marginalis mandibular nerve; PMN, platysma motor nerve.

advantages have been discussed before. Figure 3 shows an isolated partial resection of the MMN which is restored by intrafacial transfer of the PMN.

\section{CONCLUSION}

Unlike facial palsy from nonmalignant etiologies, poor long-term prognosis, advanced patients' age, and comorbidities mandate rapid restoration of facial form and function aiming at augmented quality of life to the oncologic head and neck patient. Vascularized nerve grafts, concepts of dual innervation and intrafacial nerve transfers provide adequate primary dynamic restoration of the facial nerve with low donor site morbidity, autarkic vascularity as well as suitable length, diameter and not last axonal load.

\section{REFERENCES}

1. Agrogiannis N, Rozen S, Reddy G, et al. Vastus lateralis vascularized nerve graft in facial nerve reconstruction: an anatomical cadaveric study and clinical implications. Microsurgery 2015;35:135-139

2. Owusu JA, Truong L, Kim JC. Facial nerve reconstruction with concurrent masseteric nerve transfer and cable grafting. JAMA Facial Plast Surg 2016;18:335-339

3. Bascom DA, Schaitkin BM, May M, et al. Facial nerve repair: a retrospective review. Facial Plast Surg 2000;16:309-313

4. Gidley PW, Herrera SJ, Hanasono MM, et al. The impact of radiotherapy on facial nerve repair. Laryngoscope 2010;120:19851989

5. Taylor GI, Ham FJ. The free vascularized nerve graft. A further experimental and clinical application of microvascular techniques. Plast Reconstr Surg 1976;57:413-426

6. Koshima I, Harii K. Experimental study of vascularized nerve grafts: multifactorial analyses of axonal regeneration of nerves transplanted into an acute burn wound. J Hand Surg Am 1985;10:64-72

7. Kimata Y, Sakuraba M, Hishinuma S, et al. Free vascularized nerve grafting for immediate facial nerve reconstruction. Laryngoscope 2005;115:331-336

8. Teknos TN, Nussenbaum B, Bradford CR, et al. Reconstruction of complex parotidectomy defects using the lateral arm free tissue transfer. Otolaryngol Head Neck Surg 2003;129:183-191

9. Zhu Y, Liu S, Zhou S, et al. Vascularized versus nonvascularized facial nerve grafts using a new rabbit model. Plast Reconstr Surg 2015; 135:331e-339e

10. Matsumine H, Sasaki R, Takeuchi Y, et al. Vascularized versus nonvascularized island median nerve grafts in the facial nerve regeneration and functional recovery of rats for facial nerve reconstruction study. J Reconstr Microsurg 2014;30:127-136

11. Kashiwa K, Kobayashi S, Nasu W, et al. Facial nerve reconstruction using a vascularized lateral femoral cutaneous nerve graft based on the superficial circumflex iliac artery system: an application of the inferolateral extension of the groin flap. J Reconstr Microsurg 2010;26:577-582

12. Vargel I. Impact of vascularization type on peripheral nerve microstructure. J Reconstr Microsurg 2009;25:243-253 
13. Kang SY, Old MO, Teknos TN. Lateral arm free tissue transfer for parotid reconstruction: a pictorial essay. Head Neck 2017;39:10151019

14. Iida T, Nakagawa M, Asano T, et al. Free vascularized lateral femoral cutaneous nerve graft with anterolateral thigh flap for reconstruction of facial nerve defects. J Reconstr Microsurg 2006;22:343-348

15. Brushart TM. Preferential reinnervation of motor nerves by regenerating motor axons. J Neurosci 1988;8:1026-1031

16. Brushart TM, Gerber J, Kessens P, et al. Contributions of pathway and neuron to preferential motor reinnervation. J Neurosci 1998;18:86748681

17. Hanasono MM, Skoracki RJ, Yu P. A prospective study of donor-site morbidity after anterolateral thigh fasciocutaneous and myocutaneous free flap harvest in 220 patients. Plast Reconstr Surg 2010;125:209-214

18. Collins J, Ayeni O, Thoma A. A systematic review of anterolateral thigh flap donor site morbidity. Can J Plast Surg 2012;20:17-23

19. Klebuc M, Shenaq SM. Donor nerve selection in facial reanimation surgery. Semin Plast Surg 2004;18:53-60

20. Wang W, Yang C, Li Q, et al. Masseter-to-facial nerve transfer: a highly effective technique for facial reanimation after acoustic neuroma resection. Ann Plast Surg 2014;73(suppl 1):S63-69

21. Coombs CJ, Ek EW, Wu T, et al. Masseteric-facial nerve coaptation-an alternative technique for facial nerve reinnervation. J Plast Reconstr Aesthet Surg 2009;62:1580-1588

22. Manktelow RT, Tomat LR, Zuker RM, et al. Smile reconstruction in adults with free muscle transfer innervated by the masseter motor nerve: effectiveness and cerebral adaptation. Plast Reconstr Surg 2006;118:885-899

23. Henstrom DK. Masseteric nerve use in facial reanimation. Curr Opin Otolaryngol Head Neck Surg 2014;22:284-290

24. Murphey AW, Clinkscales WB, Oyer SL. Masseteric nerve transfer for facial nerve paralysis: a systematic review and meta-analysis. JAMA Facial Plast Surg 2018;20:104-110

25. Borschel GH, Kawamura DH, Kasukurthi R, et al. The motor nerve to the masseter muscle: an anatomic and histomorphometric study to facilitate its use in facial reanimation. J Plast Reconstr Aesthet Surg 2012;65:363-366

26. Cheng A, Audolfsson T, Rodriguez-Lorenzo A, et al. A reliable anatomic approach for identification of the masseteric nerve. J Plast Reconstr Aesthet Surg 2013;66:1438-1440

27. Rodriguez-Lorenzo A, Jensson D, Weninger WJ, et al. Platysma motor nerve transfer for restoring marginal mandibular nerve function. Plast Reconstr Surg Glob Open 2016;4:e1164

28. Zhai QK, Wang XK, Tan XX, et al. Reconstruction for facial nerve defects of zygomatic or marginal mandibular branches using upper buccal or cervical branches. J Craniofac Surg 2015;26:245-247

29. Yang HM, Kim HJ, Park HW, et al. Revisiting the topographic anatomy of the marginal mandibular branch of facial nerve relating to the surgical approach. Aesthet Surg J 2016;36:977-982

30. Socolovsky MP, Bertelli JA, Masi GD, et al. Surgical anatomy of the platysma motor branch as a donor for transfer in brachial plexus repair. Surg Radiol Anat 2008;30:669-674 